\title{
Aprender Enseñando: Elaboración de Materiales Didácticos que facilitan el Aprendizaje Autónomo
}

\author{
Flor Álvarez ${ }^{*}$, Jose R. Rodríguez-Perez ${ }^{1}$, Enoc Sanz-Ablanedo ${ }^{1}$ y Marta Fernández-Martínez ${ }^{2}$ \\ (1) Universidad de León, Departamento de Tecnología Minera, Topográfica y de Estructuras, \\ Campus de Ponferrada, 24400 Ponferrada (León)-España \\ (e-mail: flor.alvarez@unileon.es, jr.rodriguez@unileon.es, esana@unileon.es) \\ (2) Universidad de León, Departamento de Psicología, Sociología y Filosofía, Campus de \\ Vegazana, 24071-León-España (e-mail: mferma@unileon.es) \\ *autor a quien debe ser dirigida la correspondencia
}

\begin{abstract}
Resumen
Este trabajo presenta el proyecto desarrollado por alumnos y profesores de tres titulaciones de ingeniería y dos centros de educación secundaria, basado en el aprendizaje autónomo y significativo de los alumnos en un entorno de aprendizaje activo. Se utilizó tecnologías de la información y la comunicación (TIC) mediante talleres supervisados y trabajo en grupos multidisciplinares donde unos enseñan a otros. Para ello, se planteó la elaboración por parte de los alumnos de ocho vídeos, como materiales didácticos, relativos a temas prácticos que se abordan en asignaturas de geomática y a la respuesta de cuestionarios. Los cuestionarios fueron analizados mediante el test de Wilkoxon Los resultados mostraron que existen diferencias estadísticamente significativas y que éstas son favorables al método presentado, por lo que se recomienda integrar en el currículo este tipo de experiencias de aprendizaje.
\end{abstract}

\section{Learning by Teaching: Development of Learning Material to Facilitate Autonomous Learning}

\begin{abstract}
This paper presents an educational method developed by students and instructors from three engineering majors and two secondary schools. During the semester students were instructed to make eight videos on practical topics covered in geomatics classes. Working on these videos enabled students to learn more about the subject while teaching others, in an active and selfdirected learning environment using Information and Communication Technologies in multidisplinary groups and supervised workshops. At the end of the project, the educational method was evaluated by using surveys completed by the students. The Wilkoxon test was then used to analyze the surveys. The results show that there were significant differences between the two teaching methods and that method presented here presents clear advantages. Therefore it is recommend to be used in higher education curricula.
\end{abstract}

Keywords: autonomous learning, learning by teaching, learning material, workshop 


\section{INTRODUCCIÓN}

Las instituciones europeas y españolas encargadas de la educación superior instan a migrar a nuevos paradigmas de enseñanza-aprendizaje, centrados en el aprendizaje autónomo durante toda la vida (LifeLong Learning, LLL) y en la consideración del estudiante como protagonista de su propio proceso de aprendizaje, dentro del marco del Espacio Europeo de Educación Superior (EEES). Sin embargo, en la actualidad, el papel protagonista es asumido en muchos casos por el profesor, mientras que la integración en la nueva filosofía del EEES requiere (i) la adecuación de los programas a la estructura ECTS (European Credit Tranfer System) de horas de trabajo del alumno, (ii) el protagonismo del alumno en el proceso de aprendizaje y (iii) el diseño de los programas de estudio basados en perfiles profesionales y académicos. Además, la sociedad actual se caracteriza por una cantidad de información infinita, dinámica y cambiante, un extensivo uso de Internet y las nuevas tecnologías, y un mercado laboral en continuo cambio, lo que requiere profesionales no sólo con conocimientos específicos y básicos, sino con destrezas para aplicarlos y resolver los problemas de un modo creativo, implicando de este modo un aprendizaje autónomo y durante toda la vida, y capacidad para trabajar en grupos multidisciplinares (Dochy et al., 2003; Ribeiro y Mizukami, 2005).

Los conceptos y estrategias, definidos en la Declaración de Bolonia y en sus posteriores desarrollos, para la construcción de un EEES, suponen un cambio en los programas educativos que deben adaptarse a un nuevo proceso formativo basado en dos puntos fundamentales: (i) aprendizaje de conocimientos específicos propios del título, y (ii) desarrollo de las habilidades y destrezas necesarias para adaptar dichos conocimientos a un campo profesional. De este modo, dentro de la formación del Ingeniero Técnico Agrícola, Ingeniero Técnico Forestal e Ingeniero Técnico en Topografía hay que atender no sólo al desarrollo y formación en una serie de competencias profesionales específicas (los conocimientos, habilidades y actitudes a desarrollar en la materia), puesto que cada titulado desempeña un puesto con un nivel de responsabilidad determinado, y para dicho desempeño necesita poseer, además, determinadas competencias transversales (relacionadas con las herramientas de aprendizaje y/o formación) siendo según el proyecto piloto "European Tuning Project", las 10 competencias más valoradas en un profesional: (1) capacidad de análisis y síntesis, (2) capacidad de aprender, (3) resolución de problemas, (4) capacidad de aplicar los conocimientos a la práctica, (5) capacidad para adaptarse a nuevas situaciones, (6) preocupación por la calidad, (7) habilidad de gestión de la información, (8) habilidad para trabajar de forma autónoma, (9) trabajo en equipo, y (10) capacidad para organizar y planificar (González y Wagenaar, 2003). Por ello los objetivos de aprendizaje deben fijarse como las metas que se pretenden en relación con las competencias, en los campos del conocimiento teórico de un campo académico (conocer y comprender), la aplicación práctica y operativa del conocimiento (saber cómo actuar), y los valores como forma de percibir y vivir (saber cómo ser).

Estas competencias se deben adquirir a lo largo de la titulación, no tanto por los contenidos de la misma, sino por el modo en que se realiza el proceso de formación, aplicando prácticas y metodologías docentes conducentes a su fomento. Los programas, por tanto, han de establecerse a partir de unos requerimientos indispensables de formación que desarrollen competencias capaces de integrar conocimiento, habilidad, actitud y destreza. Esto supone un cambio de método centrando el proceso formativo en el aprendizaje (el estudiante y su capacidad para aprender) y no en la enseñanza (el profesor). Sin embargo, la situación actual de la educación universitaria en España se basa mayoritariamente en clases presenciales de lección magistral, siendo preciso un profundo proceso de renovación metodológica, que permita dar respuesta a las nuevas demandas de la sociedad del siglo XXI (MEC, 2006).

Es por ello necesario conducir la educación superior en el área de la Ingeniería Cartográfica, Geodésica y Fotogrametría hacia un enfoque más interdisciplinario, en el que además se trabajen no sólo las competencias específicas, sino también las transversales (objetivos genéricos). En este marco surge la necesidad de emplear un método de enseñanza-aprendizaje que se ajuste a este nuevo paradigma y que considere el aprendizaje significativo y autónomo como una prioridad El documento "Propuestas para la renovación de las metodologías educativas en la universidad" (MEC, 2006), dentro de la estrategia general de renovación de las metodologías, indica que se 
debe (i) tender a la mejora del aprendizaje de los estudiantes, potenciándose las metodologías que permitan obtener en mejores condiciones los objetivos formativos y las competencias que cada disciplina tenga encomendadas en el marco de la titulación, (ii) incrementar el nivel de satisfacción y motivación de profesores y estudiantes, (iii) avanzar hacia un nuevo estilo de trabajo del profesorado, con más alternativas de actuación: clases, seminarios, tutorías, talleres, casos prácticos, trabajo con las TIC, etc., y propiciando una actuación docente cada vez más coordinada y cooperativa entre el profesorado, (iv) combinar satisfactoriamente la formación básica de los estudiantes y una mayor aproximación al ejercicio profesional real para el que se les está preparando, mediante la complementación de aprendizajes teóricos y prácticos, el trabajo sobre casos o problemas reales, el trabajo por proyectos, así como la presencia en las aulas de profesorado con experiencia profesional o de profesionales en ejercicio, y (v) aproximarnos más a los planteamientos didácticos del Espacio Europeo de Educación Superior: mayor protagonismo del estudiante en su aprendizaje, trabajo colaborativo y por competencias, adquisición de herramientas de aprendizaje, elaboración de materiales didácticos que faciliten el aprendizaje autónomo, evaluación continua, etc.

De acuerdo con estas directrices se planteó el proyecto de innovación docente "Aprender enseñando. Elaboración de materiales didácticos que faciliten el aprendizaje autónomo" durante el curso 2007/08. Se trata de un proyecto de carácter colectivo e interdisciplinario, que crea/amplía grupos de mejora docente, que se puede transferir a otros ámbitos y que se adapta a la filosofía del EEES, introduciendo innovaciones en métodos docentes y mejorando la coordinación entre disciplinas y materias. Este proyecto presenta una serie de aspectos críticos y diferenciales, plasmados a través de los siguientes objetivos específicos:

(i) Elaboración por parte de los alumnos de materiales didácticos que faciliten el aprendizaje autónomo de otros alumnos. Se trata de material audiovisual (vídeo) relativo a diferentes contenidos de carácter eminentemente práctico, que se abordan en las asignaturas relacionadas con la geomática, y que resulta difícil recoger en otro formato para que esté a disposición del alumno de forma permanente. En la actualidad no se dispone de este tipo de material, puesto que la información proporcionada por las casas comerciales no es adecuada desde el punto de vista didáctico, y material similar sólo está disponible en inglés y no se adapta a nuestro entorno de trabajo. Se propuso, por lo tanto, la realización de 8 vídeos, de una duración aproximada de 5-10 minutos, sobre los siguientes aspectos: Vídeo 1. Instrumentos topográficos: El nivel; Vídeo 2. Instrumentos topográficos: La estación total; Vídeo 3. Instrumentos topográficos: El receptor GPS; Vídeo 4. Métodos topográficos altimétricos; Vídeo 5. Métodos topográficos taquimétricos; Vídeo 6. Levantamientos topográficos con GPS; Vídeo 7. Métodos topográficos: replanteo; y Vídeo 8. Levantamientos de fotogrametría aérea: apoyo de campo.

(ii) Trabajo de los alumnos según la metodología de talleres, es decir, mediante sesiones de trabajo colaborativo en pequeños grupos multidisciplinares, formados por alumnos de las tres titulaciones implicadas: Ingeniero Técnico Forestal (esp. Explotaciones Forestales), Ingeniero Técnico Agrícola (esp. Industrias Agrarias y Alimentarias) e Ingeniero Técnico Topógrafo. Durante estos talleres los propios alumnos fueron responsables de la elaboración de los vídeos.

(iii) Actuación docente coordinada y cooperativa, de modo que los materiales didácticos generados mediante este proyecto se puedan emplear de forma directa en las siete asignaturas implicadas (Topografía, Topografía aplicada, Instrumentos Topográficos, Métodos de Levantamientos Topográficos, Levantamientos Hidrográficos, Levantamientos Batimétricos, Levantamientos fotogramétricos), y de forma indirecta en todas aquellas asignaturas (e.g. catastro, sistemas de información geográfica) y titulaciones (Ingeniero Técnico de Minas, Ingeniero de Minas, Ingeniero Agrónomo, otras especialidades de Ingeniero Técnico Agrícola) para las que resulten convenientes, dentro y fuera de la Universidad de León.

(iv) Participación del alumno en metodologías de aprendizaje activo (i.e. talleres), utilización de herramientas que fomentan el aprendizaje a lo largo de la vida (i.e. plataforma de e-learning), y trabajo con las TIC por parte de los alumnos y del profesorado. 
(v) Difusión de los materiales didácticos generados a través de la inclusión en las plataformas virtuales de aprendizaje disponibles para las asignaturas implicadas: Aula Virtual (plataforma institucional de la Universidad de León) e Ingecart (plataforma sobre Moodle del Área de Ingeniería Cartográfica, Geodésica y Fotogrametría), así como difusión a través de un canal temático de Internet.

(vi) Evaluación del método empleado (talleres) por parte de los alumnos y profesores implicados, respecto a los siguientes aspectos: la generalización (incluye todo lo referente a previsiones de futuro del método, utilidad de lo aprendido en un futuro profesional, generalización del enfoque a otras asignaturas o cursos, relación de contenidos con otras materias o con la práctica, y nuevas propuestas), los efectos y aprendizajes (hace referencia a todo lo relacionado con los tipos de conocimiento y habilidades adquiridas, las características del aprendizaje realizado), la implementación real (incluye información sobre el tiempo, el esfuerzo, la dedicación, el trabajo, exigidos por este enfoque, las dificultades, y las características y criterios de realización y evaluación) y el componente emocional (recoge los resultados relativos a satisfacción, expectativas, actitudes, motivación y clima).

\section{METODOLOGÍA}

El proyecto se desarrolló durante el curso 2007/08 en la Univ. de León (Campus de Ponferrada y se puede describir en las siguientes etapas: Participantes, Planificación, Convocatoria de los talleres, Realización de los talleres y Evaluación de los talleres, como se detalla en lo que sigue.

\section{Participantes}

El proyecto contó con la participación de 31 personas, con las siguientes funciones: (i) Veinte alumnos de segundo y tercer curso de la Escuela Superior y Técnica de Ing. Agraria: titulaciones de Ingeniero Técnico Forestal (esp. Explotaciones Forestales) e Ingeniero Técnico Agrícola (esp. Industrias Agrarias y Alimentarias), y de la Escuela Superior y Técnica de Ingenieros de Minas (titulación de Ingeniero Técnico Topógrafo), de la Univ. de León. En todas estas titulaciones es obligatorio cursar asignaturas relacionadas con la geomática y la representación del terreno. Estos alumnos son los protagonistas de este proyecto educativo, y los máximos responsables de la elaboración de los vídeos; (ii) Siete profesores del Área de Ingeniería Cartográfica, Geodésica y Fotogrametría (Departamento de Tecnología Minera, Topográfica y de Estructuras) de la Univ. de León, con docencia en las titulaciones anteriores, uno de ellos como profesor asociado.

La coordinación de cada uno de los vídeos fue asumida por uno o varios de los profesores, y estos fueron los encargados de la planificación temporal de su realización. Dos de los profesores fueron además coordinadores generales del proyecto; (iii) Una especialista en educación y psicopedagogía vinculada al departamento de Psicología, Sociología y Filosofía de la Univ. de León, encargada de la supervisión de la metodología de aprendizaje y de la evaluación del método mediante encuestas; y (iv) Profesores y alumnos de centros de formación no universitaria: un profesor del Ciclo Formativo Superior de Imagen del Instituto de Educación Secundaria (IES) Vega del Valle (Valladolid), y un profesor y un alumno del Ciclo Formativo Superior de Sonido del IES Virgen de la Encina (Ponferrada). Fueron los tutores técnicos, comunes a todos los grupos, y se encargaron de la parte audiovisual del proyecto: formación de los alumnos de la Universidad en cuestiones técnicas de imagen y sonido (planificación, grabación, montaje y edición) y supervisión de los guiones técnicos. La grabación, montaje y edición del material fue realizada en parte por cada uno de los grupos y supervisada por un alumno de segundo curso del Ciclo Formativo Superior de Sonido.

\section{Planificación}

En una primera sesión los coordinadores del proyecto y la especialista en educación informaron detalladamente de las características del método a emplear, introduciendo de este modo a todos los profesores participantes en la metodología de talleres, puesto que para la mayoría ésta fue la primera toma de contacto con la misma. Los talleres son sesiones de trabajo en pequeños grupos, 
orientadas a la consecución de un conjunto específico de resultados a partir de la participación activa de los asistentes (Exley y Dennick, 2007). Consisten en una serie de actividades vinculadas entre sí e incluidas en un marco curricular general, en ese caso la realización de un vídeo didáctico. Los contenidos mínimos a incluir en cada vídeo (como se muestra en laTabla 1), se consensuaron entre todos los profesores estableciéndose un marco de trabajo inicial.

Tabla 1. Duración y contenidos mínimos de cada vídeo

\begin{tabular}{lcl}
\hline \multicolumn{1}{c}{ Vídeo } & Duración & \multicolumn{1}{c}{ Contenidos Mínimos } \\
\hline $\begin{array}{l}\text { Instrumentos } \\
\text { topográficos: El nivel. }\end{array}$ & 5 min. & $\begin{array}{l}\text { Fundamento. Para qué sirve. Tipos. Partes del instrumento y } \\
\text { complementos necesarios. Puesta en estación. Medición básica. Errores } \\
\text { típicos: qué hacer, qué no hacer. }\end{array}$ \\
\hline $\begin{array}{l}\text { Instrumentos } \\
\text { topográficos: La } \\
\text { estación total. }\end{array}$ & 5 min. & $\begin{array}{l}\text { Fundamento. Para qué sirve. Tipos. Partes del instrumento y } \\
\text { complementos necesarios. Puesta en estación. Medición básica, ejemplo } \\
\text { de funciones especiales de la ET (medición de superficies, alturas } \\
\text { remotas...). Errores típicos: qué hacer, qué no hacer. Hacer referencia al } \\
\text { taquímetro (como precursor), diferencias con él. }\end{array}$ \\
\hline
\end{tabular}

Instrumentos topográficos: El receptor GPS.

$5 \mathrm{~min}$

Fundamento. Para qué sirve. Tipos. Partes del instrumento y complementos necesarios. Puesta en estación. Medición básica. Errores típicos: qué hacer, qué no hacer.
Métodos topográficos altimétricos.

5 min.
Objetivo de los levantamientos altimétricos. Tipos. Fases del método de nivelación (con nivel). Visualizar cómo se lleva a cabo en campo. Recomendaciones prácticas.
Métodos topográficos taquimétricos.

$5 \min$
Objetivo de los levantamientos taquimétricos con estación total. Tipos. Fases del levantamiento taquimétrico. Visualizar cómo se llevan a cabo en campo. Ventajas y limitaciones. Recomendaciones prácticas.
Levantamientos topográficos con GPS.
Objetivo de los levantamientos topográficos con GPS. Tipos de levantamientos topográficos con GPS. . Particularidades de los diferentes 5 min. tipos de levantamientos con GPS. Visualizar cómo se llevan a cabo en campo. Ventajas y limitaciones de emplear GPS. Recomendaciones prácticas
Métodos topográficos: replanteo.
Objetivo del método de replanteo. Tipos. Características generales

10 min (fases) del método de replanteo. Replanteo con nivel y con estación total: objetivo, características (particularidades), visualización de cómo se lleva a cabo en campo. Ventajas y limitaciones. Recomendaciones prácticas.
Levantamientos de fotogrametría aérea: apoyo de campo.
Necesidad del apoyo de campo para vuelos fotogramétricos.

10 min. Fundamento. Fases (planificación, ejecución y elaboración de reseñas). Visualizar cómo se lleva a cabo en campo (con GPS). Recomendaciones prácticas

Se estableció el número definitivo de alumnos adecuado para la realización de cada vídeo, de acuerdo con los contenidos establecidos, así como el perfil (preferente) de los mismos (i.e cuántos de cada titulación, si es preciso que hayan cursado alguna asignatura). Se decidió que cada uno de los 5 grupos estuviese integrado por alumnos de titulaciones y cursos diferentes, para de este modo favorecer la interdisciplinariedad y el aprendizaje de los alumnos menos expertos a partir de los más aventajados. En los grupos algunos alumnos habían cursado asignaturas afines a la temática del vídeo, y otros no, lo que implica que los objetivos de aprendizaje de cada alumno fuesen diferentes. Los vídeos se asignaron de acuerdo con su temática y duración propuesta, de 
modo que cada grupo tuviese que trabajar en dos vídeos de 5 minutos o en un vídeo de 10 minutos. De este modo cada grupo quedó constituido como una unidad de trabajo con 4 alumnos y uno o dos tutores (profesores de la Universidad de León).

\section{Convocatoria de los talleres}

Los talleres para la realización de los vídeos se convocaron la primera semana del segundo cuatrimestre del curso 2007/08 (principios de febrero). Debido a la estructura de los planes de estudio vigentes, no es posible reconocer como créditos el trabajo en los talleres por parte de los alumnos, de modo que para que este trabajo de los alumnos fuese considerado de forma efectiva, los talleres se englobaron dentro de un curso instrumental gratuito de la Universidad de León denominado "Aprender enseñando. Elaboración de materiales didácticos que faciliten el aprendizaje autónomo". De este modo los participantes en este curso fueron los participantes en los diferentes talleres para la elaboración de los vídeos durante el curso 2007/08, y su actividad se reconoció como 3 créditos de libre configuración (30 horas).

\section{Realización de los talleres}

La primera sesión de trabajo con los alumnos consistió en una reunión conjunta con los alumnos, tutores y profesores especialistas en audiovisuales, en la que se presentó la metodología a emplear. Los alumnos y tutores asistieron a una sesión teórico-práctica impartida por los profesores de los IES sobre aspectos técnicos para la grabación, edición y montaje de un vídeo, con especial hincapié en la elaboración del guión técnico, donde se recogen todas las indicaciones necesarias para poder realizar el vídeo (i.e. tipos de planos, encuadre, iluminación, secuencias, características de la toma de sonido, diálogos, efectos).

Posteriormente el trabajo se trasladó a cada grupo, donde el tutor asignado supervisó la evolución del vídeo. Los alumnos elaboraron un guión inicial de los aspectos a abordar -cuestiones en las que posteriormente ahondaron de forma autónoma, siguiendo el método de aprendizaje basado en problemas (PBL, problem-based learning)-, y discutieron la problemática del trabajo topográfico a mostrar en el vídeo, profundizando sobre la forma más correcta de ejecutar ese trabajo y los fallos más comunes, de forma que apareciesen recogidos en el vídeo. De este guión inicial se derivó un guión literario detallado, donde se indicó qué se va a decir en cada instante, y sobre el que se realizó el guión técnico definitivo. El guión literario de los vídeos fue supervisado por el tutor de cada grupo, y el guión técnico fue revisado por los profesores de los ciclos formativos superiores de Imagen y de Sonido, que se reunieron con cada grupo (alumnos y tutor) durante las tres semanas posteriores. Cada grupo trabajó sobre el guión de su vídeo, revisando las diferentes versiones en talleres en los que se reunieron todos los grupos, para discutir las diferentes propuestas, de modo que éstas se viesen enriquecidas con la aportación de otros grupos.

Una vez obtenida la versión definitiva del guión técnico, ésta se presentó al resto de grupos y tutores, para su discusión, como paso previo a la grabación. Se estableció un calendario de grabaciones, de modo que se realizaron todas durante una semana, en los emplazamientos indicados por cada grupo en el guión técnico: los exteriores del Campus y el interior de algunos laboratorios (i.e. laboratorio de fotogrametría, aula de informática). Para la grabación se empleó una cámara profesional Sony DVCAM Digital Betacam SP SX y para el montaje y edición el software Adobe ${ }^{\circledR}$ Premiere ${ }^{\circledR}$ Pro CS3. La grabación de la voz en off se realizó en el estudio del IES Virgen de la Encina. La figuración y la voz (en off o en vivo) corrieron a cargo de los alumnos de cada grupo, que asimismo colaboraron en el proceso de grabación, montaje y edición del material. El borrador de cada vídeo fue visionado y discutido por todos los grupos y tutores, en una sesión del taller, donde se hicieron recomendaciones finales para la versión definitiva de cada uno de los vídeos. Con las competencias adquiridas durante todo el proceso de elaboración del vídeo, cada grupo montó y editó el making-off (cómo se hizo) de su vídeo de forma autónoma, afianzando de este modo los conocimientos y habilidades logradas. Asimismo, se requirió que la música empleada estuviese bajo licencia Creative Commons ${ }^{\circledR}$ o equivalente, para que de este modo los alumnos se familiarizasen con temas relativos a la propiedad intelectual y las limitaciones que puede conllevar su uso. 


\section{Evaluación de los talleres}

Se realizó un visionado de los vídeos definitivos y se discutieron en grupos de debate los resultados logrados. Además, cada uno de los grupos realizó una presentación de 10-15 minutos con el "making-off" del vídeo y los problemas, en cuanto a cuestiones topográficas, surgidos durante el proceso, así como sus soluciones. Se realizó, asimismo, una evaluación de cada vídeo de forma conjunta por parte de todos los tutores y todos los participantes, destacando sus puntos fuertes y débiles, así como una serie de recomendaciones futuras. Éstas fueron recogidas por el grupo encargado del vídeo y editadas para su difusión en el informe final, que acompaña a cada vídeo.

Se evaluó el método de talleres "aprender enseñando" (AE) como herramienta de aprendizaje por parte de los alumnos, empleando 5 encuestas diferentes y analizando estadísticamente los resultados. En estas encuestas se utilizaron instrumentos escalares y de diferencial semántico, con los que se recogió información acerca de los siguientes bloques de estudio: generalización (aplicación futura, conexión con otras áreas, continuidad del método, posibilidad de generalización a otras asignaturas), efectos sobre el aprendizaje (habilidades adquiridas: expresión oral/escrita, trabajo en grupo, toma de decisiones, idiomas, autoconfianza), implementación real (información sobre el tiempo, esfuerzo, dedicación, trabajo requerido con el método de talleres, características y criterios para su evaluación/implementación) y componente emocional (satisfacción, expectativas, actitudes, motivación, ambiente), los cuales fueron establecidos en base a revisiones y estudios previos (Fernández et al., 2006; García et al., 2005). Se analizaron un total de 138 ítems, y los resultados se compararon con los resultados obtenidos en estudios previos del equipo para la lección magistral participativa (Álvarez et al., 2007). Para determinar si existen diferencias significativas entre los talleres "aprender enseñando" y el método tradicional se empleó el test no paramétrico de comparación de muestras dependientes de Wilcoxon (Norusis, 2004) al 95\% de nivel de confianza, para cada uno de los items, y agrupándolos según los aspectos indicados anteriormente.

\section{RESULTADOS Y DISCUSIÓN}

Los ocho vídeos se finalizaron tras 14 semanas de trabajo, y actualmente están disponibles en el canal temático de Internet http://es.youtube.com/user/geomaticaponferrada, al que se tiene acceso de forma gratuita. Asimismo, los vídeos y sus informes finales (puntos fuertes y débiles, recomendaciones) están disponibles en el Aula Virtual de la Universidad de León y en la plataforma de Moodle del Área de Ingeniería Cartográfica, Geodésica y Fotogrametría. Respecto al material obtenido, uno de los beneficios de este proyecto para los alumnos, es el acceso a una fuente de información complementaria de la docencia reglada (clases teóricas y prácticas), de forma que se facilita al alumno la preparación de las pruebas de evaluación. De este modo, el aprendizaje autónomo se ve incentivado mediante la disponibilidad del material en la plataforma virtual.

Además, se debe subrayar que la combinación de diferentes metodologías y herramientas didácticas en la docencia permite que alumnos con estilos de aprendizaje diferentes tengan éxito en la materia. En nuestra opinión, con este material se beneficia a aquellos alumnos cuyo estilo de aprendizaje dominante (según el modelo de Felder y Silverman, 1988) es activo (frente a reflexivo), sensitivo (frente a intuitivo), visual (frente a auditivo) y secuencial (frente a global), y que pueden encontrar dificultades extra para superar la materia empleando sólo materiales más tradicionales (clase expositiva). Además, hay numerosos estudios que consideran que los estudiantes que siguen metodologías basadas en el aprendizaje autónomo tienen un estilo de aprendizaje menos superficial, más profundo, reflexivo, autodirigido y versátil, en comparación con los que utilizan métodos tradicionales (Baker et al., 2007; Lycke et al., 2006; Tiwari et al., 2006), y que este enfoque de aprendizaje profundo suele ser un buen predictor de éxito académico, mientras que un enfoque superficial suele asociarse a peores resultados (English et al., 2004; Snelgrove, 2004; Snelgrove y Slater, 2003). 
Asimismo, el docente dispone de un recurso más para impartir la asignatura, al disponer de material didáctico que recoge procedimientos prácticos que para su realización requieren la salida a campo. Esto facilita la preparación de las asignaturas y permite que los alumnos visualicen los procesos de forma completa, incluso antes de haberlos realizado en prácticas. Los autores han hecho hincapié en la posibilidad de integrar este proyecto (talleres y empleo de material audiovisual, disponible en la plataforma de e-learning) en un entorno de aprendizaje de e-ABP (combinación del e-learning con el Aprendizaje Basado en Problemas), en marcha en algunas asignaturas del área de conocimiento desde el curso 2004/05 con muy buenos resultados y recibiendo el "Premio a la Innovación en la enseñanza" concedido por el Consejo Social de la Universidad de León en el 2007.

La investigación de la realidad, tal y como es percibida por los estudiantes, constituye una fuente de información de vital importancia si se desea comprender y mejorar el complejo proceso de aprendizaje y enseñanza (Gijbels et al., 2005; Huertas y Agudo, 2003; Struyven et al., 2005), de ahí el interés por conocer la percepción de los estudiantes en esta experiencia. Los resultados de las encuestas completadas por los alumnos muestran que existen diferencias estadísticamente significativas (nivel de confianza del 95\%) en cuanto a la implementación real entre el método "aprender enseñando" ( $A E)$ y el método tradicional, ya que los estudiantes consideran que AE fomenta más el aprender-cómo (know-how), los informes orales, el trabajo en grupo y la participación de los estudiantes, de lo que lo hace el método tradicional. Asimismo, los alumnos consideran que la comunicación alumno-profesor fuera de la clase es más importante en AE, así como la evaluación frecuente del proceso de aprendizaje y el uso de nuevas tecnologías y recursos. Los resultados de la encuesta sobre actitudes/sentimientos de los alumnos indican que existen diferencias significativas entre los métodos, y que las reacciones/sentimientos de los alumnos son más positivos en el AE. Además, los resultados indican que existen diferencias en los efectos sobre el aprendizaje, i.e. los alumnos perciben una mejoría en sus habilidades mayor en el caso del AE, siendo especialmente efectivo en las habilidades sociales y comunicativas, el desarrollo de pensamiento crítico y el aumento de la responsabilidad. En cuanto al componente emocional, los alumnos encuentran el AE más interesante, entretenido y motivador, además de permitirles el aprendizaje autónomo y hacerles sentir orgullosos de su trabajo. Otros aspectos considerados como muy positivos por el alumno en relación con AE fueron: el trabajo cooperativo, la participación activa y la integración de competencias transversales. En relación con la generalización, los alumnos tuvieron una actitud significativamente más positiva respecto al AE, y recomendaron su implementación en otras áreas. Estos resultados concuerdan con los obtenidos por Fernández et al. (2006), que realizó una revisión sobre la evaluación y comparación de los métodos de enseñanza activa (e.g. aprendizaje basado en problemas) con el enfoque tradicional (lección magistral), considerando sus efectos sobre estos aspectos, y concluyendo que estas metodologías activas son más positivas en la mayoría de los casos.

Los docentes participantes han destacado los beneficios derivados de la coordinación y cooperación desde diferentes asignaturas, de modo que el trabajo conjunto redunda en una mejor conexión entre los contenidos de las asignaturas y en el fomento de un clima de cooperación entre el profesorado, idea señalada en diversos estudios (Blumberg, 2003; Streichert et al., 2005). Del mismo modo, el contacto con profesionales en ejercicio (i.e. profesor asociado) implica de forma indirecta una actualización del propio profesor en cuanto a técnicas, métodos e instrumentos. Se ha señalado como dificultad el conseguir que los alumnos trabajasen realmente en equipo, puesto que no están habituados a hacerlo de una forma tan intensa y continuada en el tiempo. Esta misma dificultad ha sido destacada por Mesa et al. (2008), al emplear el método de aprendizaje basado en problemas en otras asignaturas de ingeniería. Asimismo, el desarrollo de este proyecto ha favorecido la relación entre alumnos y profesores, al trabajar de forma conjunta en grupos reducidos y supervisados, con un seguimiento y atención más cercana que durante las clases convencionales.

\section{CONCLUSIONES}

La realización de vídeos didácticos por parte de los alumnos mediante la organización de talleres y fundamentándose en aprender enseñando, fomenta el aprendizaje activo y profundo: los estudiantes interaccionan con materiales de aprendizaje, relacionan conceptos con actividades de 
la vida diaria, mejorando así su comprensión, la retención y el desarrollo de habilidades de aprendizaje para toda la vida. El conocimiento de diferentes disciplinas se integra para dar solución al problema sobre el cual se está trabajando (la materia tratada en el vídeo), de tal modo que el aprendizaje no es fragmentario sino integral y dinámico facilitándose así la adquisición de un currículo integrado, la activación de conocimientos previos y el aprendizaje significativo.

Las competencias generales y específicas, el aprender a aprender y el aprendizaje a lo largo de la vida, mejoran más empleando EA que con la lección magistral. El alumno mejora respecto a sus habilidades cognoscitivas, capacidades metodológicas (cómo organizarse, tomar decisiones, resolver problemas e imprevistos), destrezas tecnológicas (de computación, TIC y gerencia de información), y destrezas lingüísticas (comunicación oral y escrita, empleo de lenguaje técnico con rigor). Además, mejora en sus habilidades criticas y de autocrítica, y en la capacidad de transmitir sus opiniones, así como en las destrezas sociales relativas a habilidades interpersonales que facilitan la interacción social y la cooperación (trabajo en equipo).

En un currículo de educación superior dispuesto de manera constructiva, con una especificación de programa bien definida, todas las experiencias de aprendizaje (clases magistrales, seminarios, sesiones prácticas, aprendizaje autodirigido y sesiones de enseñanza en pequeños grupos) deben constituir un todo coherente e integrado. Por todo ello, se recomienda integrar en el currículo experiencias de aprendizaje como la llevada a cabo en este proyecto, basada en aprender enseñando y en el trabajo en grupos interdisciplinares mediante talleres supervisados.

\section{AGRADECIMIENTOS}

Este proyecto ha sido financiado por el Plan de Ayuda a la Innovación Docente del curso 2007/08 de la Universidad León (España).

\section{REFERENCIAS}

Álvarez, M. F. y otros cuatro autores; Aprendizaje activo y autónomo en Ingeniería: el método e$A B P$. Evaluación de los efectos sobre el aprendizaje, implementación real, generalización y componente emocional. Actas de las I Jornadas Internacionales UPM sobre Innovación Educativa y Convergencia Europea. Universidad Politécnica, Madrid, 12-14 de diciembre (2007).

Baker, C.M., D.J. Pesut, A.M. McDaniel y M.L Fisher; Evaluating the impact of problem-based learning on learning styles of master's students in nursing administration, Journal of Professional Nursing: 23(4), 214-219 (2007).

Blumberg, P.; Multidimensional outcome considerations in assessing the efficacy of medical educational programs, Teaching and Learning in Medicine: 15(3), 210-214 (2003).

Dochy, F., M. Segers, P. Van den Bossche y D. Gijbels, Effects of problem-based learning: a meta-analysis, Learning and Instruction: 13(5), 533-568 (2003).

English, L., P. Luckett y R. Mladenovic; Encouraging a deep approach to learning through curriculum design, Accounting Education: 13(4), 461-488 (2004).

Exley, K. y R. Dennick; Enseñanza en pequeños grupos en educación superior. Tutorías, seminarios y otros agrupamientos. Ed. Narcea, Madrid (2007).

Felder, R.M. y L.K. Silverman; Learning and Teaching Styles in Engineering Education, Engr. Education: 78(7), 674-681 (1988).

Fernández, M. otros cuatro autores; El aprendizaje basado en problemas: revisión de estudios empíricos internacionales, Revista de Educación: 341, 397-418 (2006).

García, J.N., A.M. de Caso, R. Fidalgo y O. Arias; La evaluación de prácticas universitarias y su aplicación en un enfoque innovador, Revista de Educación: 337, 295-325 (2005). 
Gijbels, D., G. van de Watering y F. Dochy; Integrating assessment tasks in a problem-based learning environment, Assessment \& Evaluation in Higher Education: 30(1), 73-86 (2005)

González, J. y R. Wagenaar (eds.); Tuning Educational Structures in Europe. Informe Final.

Bilbao: Universidad de Deusto, RGM, S.A. publicadores, Bilbao-España (2003).

Huertas, J.A. y R. Agudo; Concepciones de los estudiantes universitarios sobre la motivación. En C. Monereo, \& J. I. Pozo (Eds.), La universidad ante la nueva cultura educativa. Enseñar y aprender para la autonomía. (pp. 45-62). Ed. Síntesis, Madrid (2003).

Lycke, K.H., P. Grottum y H.I. Stromso; Student learning strategies, mental models and learning outcomes in problem-based and traditional curricula in medicine, Medical Teacher: 28(8), 717-722 (2006)

Mesa, J.M., J.V. Álvarez, J.M. Villanueva y F.J. de Cos; Actualización de Métodos de EnseñanzaAprendizaje en Asignaturas de Dirección de Proyectos de Ingeniería, Form. Univ.: 1(4), 23-28 (2008)

MEC; Propuestas para la renovación de las metodologías educativas en la universidad, Ministerio De Educación Y Ciencia (MEC), Consejo de Coordinación Universitaria; , Secretaría General Técnica, Edigrafos, S. A. (2006).

Norusis, M.; SPSS 13.0 Statistical procedures companion, Prentice Hall, Inc., Upper Saddle-River, New Jersey, USA (2004).

Ribeiro, L.R. y M.G. Mizukami; Problem-based learning: a student evaluation of an implementation in postgraduate engineering education, European J. Eng. Educ.: 30(1), 137-149 (2005) Snelgrove, S.R.; Approaches to learning of student nurses, Nurse Education Today: 24, 605-614 (2004).

Snelgrove, S.R. y J. Slater; Approaches to learning: psychometric testing of a study process questionnaire, Journal of Advanced Nursing: 43(5), 496-505 (2003).

Streichert, L.C. y otros cinco autores; Using problem-based learning as a strategy for crossdiscipline emergency preparedness training, Journal of Public Health Managemet and Practice: November (Suppl), 95-99 (2005).

Struyven, K., F. Dochy y S. Janssens; Students' perceptions about evaluation and assessment in higher education: a review, Assessment \& Evaluation in Higher Education: 30(4), 325-341 (2005).

Tiwari, A. y otros seis autores, The effect of problem-based learning on students' approaches to learning in the context of clinical nursing education, Nurse Education Today: 26, 430-438 (2006). 